\title{
The Relationship Between Performance and Asymmetries in Different Multidirectional Sprint Tests in Soccer Players
}

\author{
by \\ Alberto Fílter ${ }^{1,2}$, Vicente Beltrán-Garrido ${ }^{3}$, Thomas Dos'Santos ${ }^{1,4}$, \\ Daniel Romero-Rodríguez ${ }^{5,6}$, Bernardo Requena ${ }^{1}$, Irineu Loturco ${ }^{1,7,8,9}$, \\ Marc Madruga-Parera ${ }^{10,11,12}$
}

Practitioners usually include change of direction (COD) and linear speed measurements in the testing batteries of soccer players; however, despite being a commonly occurring action, curve sprint (CS) ability is rarely assessed in soccer. The aims of this study were to analyze the association between linear sprint, CS, and COD speed performances, and compare the association and direction of asymmetries between these skills. Thirty-three male soccer players performed linear sprint $(17 \mathrm{~m}), \mathrm{CS}(17 \mathrm{~m})$, and COD-90 speed tests (COD [8.5 $8.5 \mathrm{~m}])$. Our main findings were (a) a large relationship between linear and multidirectional tasks (COD-90 and CS tests) ( $r=$ from 0.6 to $0.64, p$ $<0.05$ ), (b) a moderate relationship between CS and COD-90 $0^{\circ}$ tests ( $r=$ from 0.33 to 0.41, $p<0.05$ ), with a certain opposite tendency (higher relationships between opposing directions [CurvelEFT - CODRIGHT; $r=0.41$ ] than between equal directions [CUrveLEFT - CODLEFT; $r=0.33$ ]), and (c) no relationship $(p>0.05)$ between COD and CS asymmetries, with opposing directional dominance in $\sim 70 \%$ of players (e.g., curve left and COD right dominance). These results indicate that performance in linear sprints is strongly related to performance in multidirectional trajectories, whereas CS and COD-90 seem to be more independent actions. Additionally, the direction of asymmetry or dominance is generally opposite between the non-linear tasks measured.

Key words: speed assessment, curvilinear speed, change of direction.

\section{Introduction}

In soccer, a wide spectrum of different trajectories during acceleration maneuvers is usually performed (i.e., linear sprints, curve sprints [CS], and change of direction [COD] tasks)
(Jeffreys et al., 2018). Results of motion analysis demonstrate that soccer players perform $\sim 700$ turns during official matches, most of which are at $0-90^{\circ}$ (Bloomfield et al., 2007), and the vast majority of the sprints performed in soccer are not

\footnotetext{
1 - FSI Sport Research Lab, Granada, Spain.

2 - Research Group Physical Activity, Health and Sport CTS-948, University of Pablo de Olavide, Seville, Spain.

3 - EUSES Health and Sport Science School, Rovira i Virgili University, Tarragona, Spain.

4 - Department of Sport and Exercise Sciences, Musculoskeletal Science and Sports Medicine Research Centre, Manchester Metropolitan University, Manchester, United Kingdom.

5 - FC Barcelona First Team, Sport Performance Area, Barcelona, Spain.

6 - International University of Catalonia, Physical Therapy Department, Barcelona, Spain.

7 - NAR - Nucleus of High Performance in Sport, Sao Paulo, Brazil.

8 - Department of Human Movement Sciences, Federal University of Sao Paulo, Sao Paulo, Brazil.

9 - University of South Wales, Pontypridd, Wales, United Kingdom.

10 - ReQ, Return to Play and Sports Training Center, Barcelona, Spain.

11 - FC Barcelona Second Team, Sport Performance Area, Barcelona, Spain.

12 - University School of Health and Sport (EUSES), University of Girona, Girona, Spain.
} 
purely linear, but with a degree of curvature $(\sim 85 \%)$ used to evade, track, or draw an opponent (Fitzpatrick et al., 2019; Caldbeck, 2020). Exposure to speed training programs designed according to specific game demands (the concept of "gamespeed") is crucial for any player wishing to maximize soccer performance (Jeffreys et al., 2018). In this sense, players in different positions could benefit from more specific conditioning programs and testing routines (Bloomfield et al., 2007; Maszczyk et al., 2011; Fitzpatrick et al., 2019). This means that practitioners should consider individual multidirectional sprint tests which are specific to the movement demands of soccer to detect actual (and effective) changes in speed-related performance.

The inclusion of COD speed and maneuverability measurements is recommended in testing batteries for soccer players (Arcos et al., 2020; Cuthbert et al., 2019; Dos'Santos et al., 2018, 2019; Loturco et al., 2020). As such, a variety of COD speed measurements have been used by practitioners to assess multidirectional speed ability in soccer players, (i.e., T-test, COD90 ${ }^{\circ}, 5-0-5$ agility test) (Brughelli et al., 2008; Turner et al., 2011), but none of them includes curvilinear sprints or assesses the isolated ability to sprint over curvilinear trajectories. From a directional approach, a CS test is considered a hybridization between a linear sprint and a COD drill (i.e., COD: a test that contains a clear lateral foot plant to initiate the COD) (Fílter et al., 2019; Nimphius, 2014). In terms of relationships, some of correlations between COD and straight speed performances would be described as moderate $(r$ $=0.3-0.5)$ in different level and sport populations (i.e., college students, Australian football players) and different types of COD tests (i.e., T-test, 20-m COD [three 90 CODs]) (Brughelli et al., 2008). In fact, the magnitude of correlations seems to be influenced by the COD characteristics (i.e., angles and numbers) (Rouissi et al., 2016; Young et al., 2002), and a sport population (i.e., soccer, volleyball, basketball, handball) (Šimonek et al., 2017). Specifically, in soccer players, moderate correlations $(\mathrm{r}=0.35)$ have been observed between straight running and Zigzag COD test $(4 \times 5$ COD with $100^{\circ}$ ) completion times (Little and Williams, 2005); however, no significant correlations have been found between other COD tests (i.e., 505 and T-test) and linear sprint performance (10-, 20-, 30m) (Çınarlı et al., 2018).

In this regard, a similar significant association has been observed between linear and curvilinear speed (Fílter et al., 2019). In fact, despite being considered to be independent physical qualities (i.e., $35 \%$ shared variance), linear and CS actions exhibit a large interrelationship $(\mathrm{r}=0.58)$, which means that at least part of a given increase or decrease in a specific scale is common between these capacities. Nonetheless, to date, no study has directly examined the relationship between the COD-90 speed test and curved sprinting; two apparently similar non-linear actions.

Given the unpredictable nature of soccer, being equally fast at changing direction during COD maneuvers and efficient at performing both right (clockwise) and left (anti-clockwise) curvilinear sprints would be beneficial from a performance perspective. In this sense, not only performance association is relevant, but also analyzing the magnitudes and directions of interlimb asymmetries, which would aid understanding of the similarities between both tasks. Recently, there has been a rise in the number of studies investigating the magnitude and direction of interlimb functional asymmetries (i.e., assessed, for example, in vertical and horizontal jumps, or COD tasks) and asymmetries in some specific neuromuscular qualities (Dos'Santos et al., 2019; Madruga-Parera et al., 2019, 2020; Raya-González et al., 2020). Nevertheless, despite being a frequently used action (Caldbeck, 2020; Fitzpatrick et al., 2019), none of the studies considered curvilinear sprints. For example, in a study with female youth netball players, Dos'Santos et al (2019) reported asymmetries of $\sim 2 \%$ and $\sim 12 \%$ for $5-0-5$ test completion times and COD deficits (time difference from linear sprints), respectively. The authors concluded that quantifying asymmetries based on COD completion times may produce a misinterpretation (i.e., lower percentage imbalances) of an athlete's symmetry in COD ability, which could mask deficiencies in COD performance (Dos'Santos et al., 2019). However, this deficit-based analysis cannot be performed between curved and linear paths due to possible similar records (Fílter et al., 2019, 2020; Ohnuma et al., 2018). Another study conducted with elite youth soccer players found asymmetries of $4.6 \%$ 
and $3.02 \%$ for both COD- $90^{\circ}$ tests $(5+5$ and $10+$ $10 \mathrm{~m}$, respectively), highlighting that asymmetries vary across commonly used tests without significant interrelationships (Bishop, 2020; RayaGonzález et al., 2020). In this line, knowing the differences between clockwise and anti-clockwise completion times would contribute to obtaining asymmetry thresholds in curvilinear sprints, and comparing these values with the COD- $90^{\circ}$ measurement.

Given the limited research investigating curved sprinting in soccer players, and the lack of investigations regarding the relationship between COD speed and CS abilities, the aims of this study were to: (a) analyze the relationships between linear sprint, CS, and COD speed performances, and (b) compare the associations and direction of asymmetries between CS and COD speed tests. We hypothesized that (a) limited and nonmeaningful associations would be observed between CS, linear sprint, and COD speed performance, and (b) limited and non-meaningful associations would be detected between CS and COD speed asymmetries.

\section{Methods}

\section{Participants}

Thirty-three semi-professionals (3rd Spanish division) male soccer players volunteered to participate in this study (age: $23.4 \pm 0.8$ years; body height: $1.78 \pm 0.50 \mathrm{~m}$; body mass: $74.2 \pm 8.4$ $\mathrm{kg})$. Subjects were excluded if they presented any injury at the time of testing. Tests were completed during the third month of the competition period (nine-month season). Written consent forms were obtained from all participants. The study was conducted in accordance with the Declaration of Helsinki and was approved by the Catalan Sports Council Ethics Committee.

\section{Design and Procedures}

Following a cross-sectional design, a sample of soccer players performed $90^{\circ}$-change of direction, CS, and linear sprint tests of $17 \mathrm{~m}$ each. Accordingly, interlimb asymmetries in CS and COD speed tests were examined. Correlational analyses were also used to determine the relationships between the multiple tests and the magnitudes of asymmetries between COD speed and CS assessments.

Participants arrived at our facilities prior to the first training session of the week having been instructed not to eat for the preceding 2 hours and avoid caffeine consumption for at least 24 hours before the tests. All athletes were familiarized with the testing procedures. Participants were tested on two different days, separated by 72 hours. Day 1 consisted of a $17 \mathrm{~m}$ linear sprint and the $90^{\circ} \mathrm{COD}$ speed assessment. On day 2, players performed the $17 \mathrm{~m}$ CS test.

Each participant completed a specific warm up consisting of five minutes of light jogging and dynamic stretches for the lower body (such as multi-directional lunges, inchworms, bodyweight squats, and sprint drills). Upon completion, three practice trials were provided for each test and participants were instructed to perform them at 75,90 and $100 \%$ of their perceived maximal effort. Three minute rest intervals were allowed between all trials. The tests were executed on synthetic field turf.

To perform the tests players began in a staggered two-point stance $0.5 \mathrm{~m}$ behind the start photocells. Completion times were measured via photocells (Witty, Microgate, Bolzano, Italy). The photocell height was individually adjusted to match each athlete's ground-to-hip height. The fastest time of the three trials was used for further analysis.

Measures

Linear Sprint test: Participants were required to perform a linear sprint for a total distance of $17 \mathrm{~m}$ as fast as possible, in line with previous research (Fílter et al., 2019). Each trial was separated by a 3-minute rest interval.

Change of direction speed test: For the COD test we used the recommendations of Maloney et al. (2016) who reported intraclass correlation coefficients (ICCs) of 0.95 and 0.97 (within- and between-session ICCs, respectively). Both sides of the COD (right direction and left direction) were assessed in a single $90^{\circ} \mathrm{COD}$ for a total distance of $17 \mathrm{~m}$ (Figure 1). A guideline was used as a reference and the path was delimited with cones to avoid curvilinear paths. Three successful trials for each side were performed. The trial was considered successful if the player performed a clear lateral foot plant at the turning point. Each trial was separated by a 3 minute rest interval.

Curve sprint test: For the CS assessment we used the recommendations of a previous study (Fílter et al., 2019) which showed a reliable 
standardized test performed on the penalty arc line as a guideline (Figure 2). Left (anti-clockwise) and right (clockwise) curves were assessed. In order to unify terminology with COD research (Maloney et al., 2016; Dos'Santos et al., 2019; Madruga-Parera et al., 2019), a dominant and a non-dominant curve (fastest and slowest side in completion time, respectively) were used. Players were instructed to follow the penalty arc line and performed three trials in each direction in randomized order. If the player did not run within the penalty arc lines, the trial was disqualified and repeated following the rest period. Regarding reliability, the coefficient of variation $(\mathrm{CV})$ and the intraclass correlation coefficient (ICC) were calculated, with $2 \%$ and 0.87 for each coefficient, respectively. Each trial was separated by a 3 minutes rest interval.

\section{Statistical analyses}

Inter-limb asymmetries in COD and CS tests were quantified as a percentage difference between limbs using the formula: (100/(maximum value $\left.)^{*}(\text { minimum value })^{*}-1+100\right)$, as proposed by Bishop et al. (2018). In order to determine the direction of asymmetry, an "IF function" was added to the end of the formula in Microsoft Excel: ${ }^{*}$ IF (left<right, 1, -1) (Bishop et al., 2020).

Analyses were performed using JASP for Mac (version 0.11.2; JASP Team (2019), University of Amsterdam, the Netherlands) and IBM SPSS Statistics for Mac (v.25, IBM, New York). Normal distribution of the data was checked using the Shapiro-Wilk test. Data not following a normal distribution were log-transformed (Field, 2013) before further analysis.

To examine performance differences between directions, paired-samples t-tests were used. Effect size (Cohen's $d_{z}$ ) calculations were performed on pairwise comparisons, which were computed using the formula $d_{z}=t / \sqrt{ }(n)$ (Lakens, 2013). Effect sizes were interpreted as: $<0.2=$ trivial; $0.2-0.6=$ small; $0.6-1.2=$ moderate $; 1.2-2.0=$ large $>2.0=$ very large (Hopkins et al., 2009).

To assess the relationships between the performance test scores and between asymmetry scores of each test, Pearson's product moment correlation coefficients $(r)$ were computed. The correlation coefficients were interpreted as follows: $r=0.0-0.1=$ trivial, $0.11-0.3=$ small, $0.31-$ $0.5=$ moderate, $0.51-0.7=$ large, $0.71-0.9=$ very large, and 0.91-1.00 nearly perfect (Hopkins et al.,
2009). The determination coefficient $\left(\mathrm{r}^{2}\right)$ was calculated to establish the degree of shared variance between tasks. The level of significance was set at 0.05 for all tests.

\section{Results}

Mean and asymmetry scores and reliability data are presented in Table 1.

The relationships of performance times between assessments are presented in Table 2 . The linear sprint speed demonstrated large relationships with all performance test scores $(r=$ $0.60-0.64, p<0.001)$. The CODleft variable showed a very large relationship with COD $=0.81, p<0.001)$ and a moderate relationship with Curveright $(r=0.38, p<0.05)$. The CODright variable presented moderate relationships with Curveleft $(r=0.41, p<0.05)$ and Curveright $(r=$ $0.35, p<0.05)$. The Curveleft showed a large relationship with Curveright $(r=0.66, p<0.001)$.

The coefficients of determination revealed that shared variance between variables was limited (Table 2). Linear sprint speed shared between 36 and $41 \%$ of variance with COD speed and CS measurements. In addition, the shared variance reduced between the COD speed and CS measurements, with a shared variance between 11 and $16 \%$.

No significant relationship was reported between COD speed and CS speed asymmetry data $(\mathrm{r}=-0.05 ; p>0.05)$. In addition, Figure 4 shows that $\sim 70 \%$ of participants presented asymmetries in opposite directions between the CS and the COD tasks.

\section{Discussion}

The main findings of this study were: (a) the multidirectional measurements (assessed via the $90^{\circ}$ COD speed and CS tests) were largely correlated with linear sprint tests, and (b) a moderate relationship was observed between multidirectional tests (COD speed and CS tests) with a certain "opposite tendency" (a higher relationship between opposing directions [Curveleft - CODRIGHT; $r=0.41$ ] than between equal directions [Curveleft - CODleft; $r=0.33$ ]), and c) there were no meaningful relationships between COD speed and CS asymmetries. This indicates that direction dominance during COD tasks does not necessarily imply the same 
directional dominance during curved sprinting (and vice versa). The data of the present study support previous studies in relation to the association between linear speed and CS performances in semi-professional soccer players (Fílter et al., 2019), and the association between
COD and linear sprinting (Little and Williams, 2005); nevertheless, this is the first study to examine the relationship between COD (via the $90^{\circ}$ COD speed test) and CS speed in semiprofessional soccer players.

Table 1

Interlimb neuromuscular test scores and reliability data for each test.

\begin{tabular}{|c|c|c|c|c|}
\hline Test & Mean \pm SD (s) & $\mathrm{d}_{\mathrm{z}}(95 \% \mathrm{CI})$ & CV $(95 \%$ CI) (\%) & ICC (95\% CI) \\
\hline Sprint $_{(\mathrm{s})}$ & $2.69 \pm 0.10$ & - & $1.43(1.09,1.76)$ & $0.93(0.88,0.96)$ \\
\hline CODleft(s) & $3.22 \pm 0.18$ & & $1.88(1.52,2.25)$ & $0.95(0.89,0.98)$ \\
\hline $\begin{array}{l}\text { CODRIGHT } \\
\text { (s) }\end{array}$ & $3.24 \pm 0.16$ & $-0.12(-0.46,0.23)$ & $2.29(1.56,3.02)$ & $0.87(0.76,0.93)$ \\
\hline CODD (s) & $3.19 \pm 0.16^{*}$ & $-1.19(-1.63-0.74)$ & & \\
\hline $\mathrm{CODND}_{\mathrm{N}(\mathrm{s})}$ & $3.27 \pm 0.17$ & & & \\
\hline CODASY(\%) & $2.33 \pm 1.67$ & & & \\
\hline CSLefF(s) & $2.68 \pm 0.11$ & & $2.06(1.59,2.54)$ & $0.88(0.79,0.94)$ \\
\hline CSRIGHT(s) & $2.67 \pm 0.10$ & $0.20(-0.15,0.54)$ & $2.04(1.53,2.55)$ & $0.86(0.75,0.93)$ \\
\hline $\mathrm{CS}_{\mathrm{D}(\mathrm{s})}$ & $2.62 \pm 0.12^{*}$ & & & \\
\hline $\mathrm{CS}_{\mathrm{ND}(\mathrm{s})}$ & $2.72 \pm 0.13$ & $-1.06(-1.48,-0.63)$ & & \\
\hline $\mathrm{CS}_{\mathrm{ASY}(\%)}$ & $3.41 \pm 2.37$ & & & \\
\hline
\end{tabular}

${ }^{*} p<0.05$, compared with non-dominant; ASY: Asymmetries; $C I=$ confidence intervals; $C V=$ coefficient of variation; $C O D=$ change of direction; $C S=$ Curve sprint of $17 m ; d=$ Cohen's $d_{z} ; D=$ Dominant (fastest side); ICC: intraclass correlation coefficient; $N D=$ Non-dominant (slowest side); Sprint $=$ linear sprint test of 17 m; s: Seconds.

Table 2

Relationships [percentage of shared variance] (Pearson's coefficient of correlation) between performance score tests with $95 \%$ confidence intervals.

\begin{tabular}{|c|c|c|c|c|}
\hline & Sprint & CODleft & CODRIGHT & CSLeft \\
\hline & $0.64[41 \%]$ & & & \\
\hline \multirow[t]{2}{*}{ CODleft } & $(0.81,0.38)^{* * *}$ & - & - & - \\
\hline & $0.60[36 \%]$ & $0.81[66 \%]$ & & \\
\hline \multirow[t]{2}{*}{ CODRIGHT } & $(0.78,0.33)^{* * *}$ & $(0.90,0.64)^{* * *}$ & - & - \\
\hline & 0.64 [41\%] & 0.33 [11\%] & $0.41[16 \%]$ & \\
\hline \multirow[t]{2}{*}{ CS Leff } & $(0.81,0.38)^{* * *}$ & $(0.61,-0.01)$ & $(0.66,0.08)^{*}$ & - \\
\hline & $0.64[41 \%]$ & 0.38 [14\%] & 0.35 [12\%] & $0.66[44 \%]$ \\
\hline CSRIGHT & $(0.81,0.38)^{* * *}$ & $(0.64,0.04)^{*}$ & $(0.62,0.01)^{*}$ & $(0.82,0.41)^{* * *}$ \\
\hline
\end{tabular}

${ }^{*} p<.05 ;{ }^{* *} p<.01 ;{ }^{* * *} p<.001$, compared with another test cited; $C O D=$ change of direction;

$C S=$ Curve sprint of $17 \mathrm{~m}$; Sprint $=$ linear sprint test of $17 \mathrm{~m}$. 


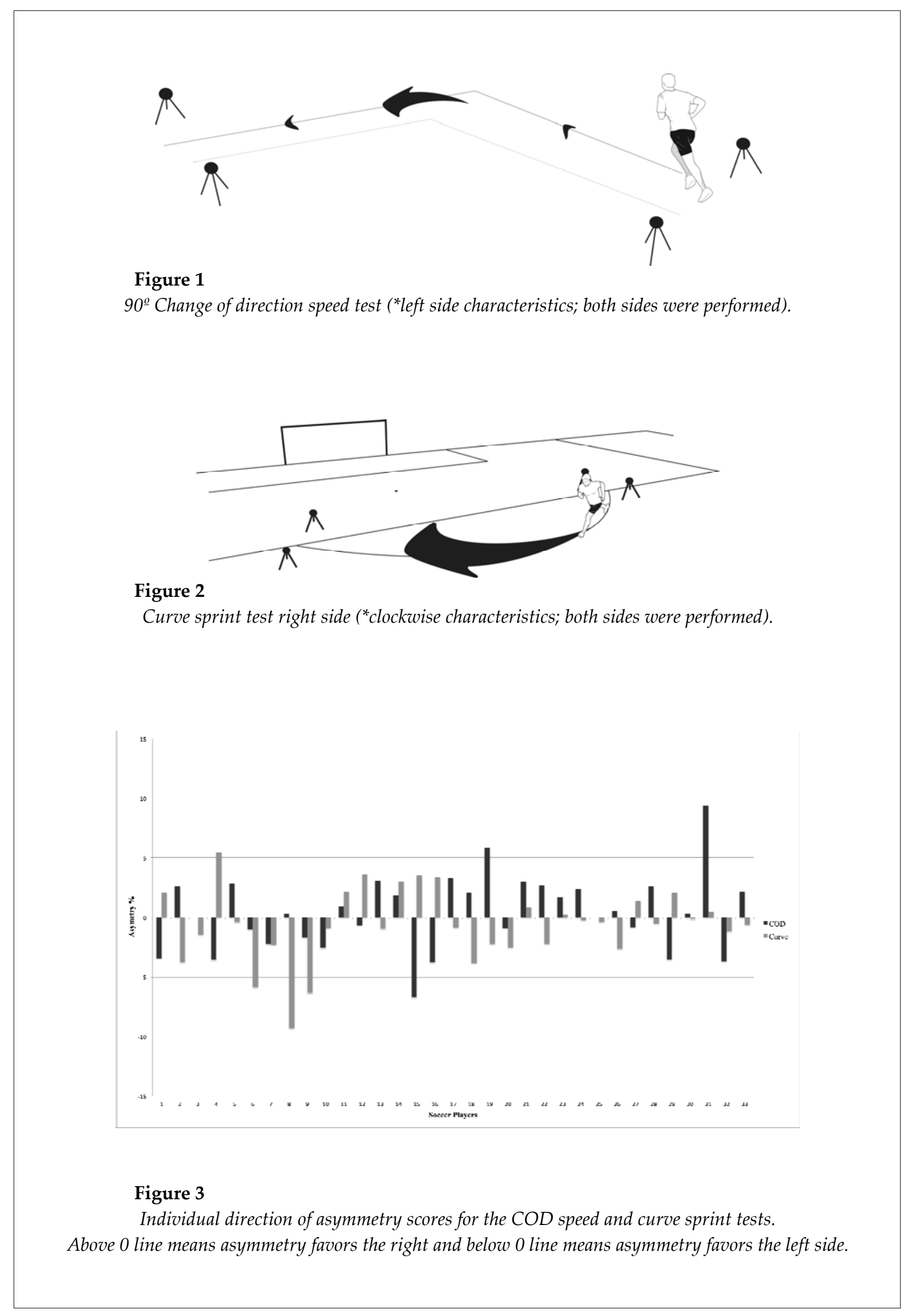


Filter et al. (2019) examined the association between linear speed and CS (35\% shared variance), with similar results to those reported in this work $(\mathrm{r}=\sim 0.58)$. More recently, Loturco et al. (2020) reported large to very large relationships (0.67-0.76) between linear and CS velocities in elite young soccer players, suggesting that these differences may be related to distinct sample characteristics (i.e., semi-professional versus elite youth soccer players). In addition, the large relationship between linear and multidirectional tasks (COD-90 $0^{\circ}$ and CS test [r from 0.6 to 0.64 ]) could be explained by common elements between these respective short all-out efforts (i.e., player's acceleration capacity), and the reduced shared variance (i.e., $\leq 40 \%$ ) as a consequence of their independent and particular features (e.g., specific cutting and arced maneuvers) (Young et al., 2001). This issue certainly deserves further investigation.

Although researchers have examined the relationship between linear speed and COD speed performance in soccer players (Buttifant et al., 1999; Little and Williams, 2005; Suarez-Arrones et al., 2020), only one study has examined the associations between COD and CS speed in semiprofessional soccer players, reporting "large" relationships between these variables (from 0.54 to 0.60 ) (Freitas et al., 2020). However, unlike this work, that study assessed COD maneuverability for both sides during multiple directional changes within the same task (i.e., Zigzag test), without calculating and considering inter-limb asymmetries in COD and CS measurements in its rationale.

The novel finding of this study is the limited association between single $90^{\circ} \mathrm{COD}$ speed and CS tests, with a shared variance of $~ 11 \%$. Thomas and Nelson (1990) state that "when common variance between two variables is less than $50 \%$, this indicates that they are specific or somewhat independent in nature". Thus, although a turning degree can affect the magnitude of the relationship between non-linear actions (Brughelli et al., 2008; Young et al., 2001) (e.g., a higher ratio between sharp COD and curvilinear sprint), COD speed (assessed via the $90^{\circ}$ COD speed test), and CS, at least for this population of semiprofessional soccer players, they may be considered independent skills and, as a consequence, should be evaluated and likely trained separately.

From a training perspective, a previous study (Young et al., 2001) examined the effect of linear sprint training on COD speed, concluding that linear sprint training has limited ability to transfer to COD performance. However, association does not necessarily imply causation, and this cross-sectional study requires longitudinal data to examine the effects of increasing linear sprint velocity on COD speed and CS performance. Although this study focused on the physical components of multidirectional sprints, the role of perceptual skills, such as reacting to the visual presence of the ball and opponents, could also be considered in further research.

Trivial and non-significant relationships between COD asymmetries and CS asymmetries were found, confirming the task-dependent nature of inter-limb asymmetries. When individual magnitude and directional asymmetry percentages were examined (Figure 4 ), $\sim 70 \%$ of soccer players (22/33) were categorized inversely in terms of the direction of asymmetries when using COD or CS tests. In summary, this means that players who had asymmetries for one side in the COD test presented an opposite direction in the CS test.

Based on the findings reported in previous studies, it seems logical that asymmetries between CS and COD-90 $0^{\circ}$ will exhibit opposite directionality in most soccer players analyzed. In fact, most compelling evidence is in favor of the greater contribution of the outside leg during COD tasks (Young et al., 2002), and the inside leg during curvilinear sprints (Chang and Kram, 2007; Filter et al., 2020). Young et al. (2002) concluded that the outside leg would exert more influence than the inside leg in turning movements if the movements require a "clear lateral push-off action" (i.e., COD-90). Conversely, during maximum effort, a reduction in peak resultant ground reaction force by the inside leg plays a significant role in limiting speed during curvilinear paths (Chang and Kram, 2007; Filter et al., 2020). Furthermore, this study obtained a higher relationship between COD and CS on opposite sides than on equal sides (Table 2). The present research provides findings suggesting that COD (assessed via the $90^{\circ}$ COD speed test) and CS abilities are opposite qualities 
in terms of directional dominance.

The $90^{\circ}$ COD speed assessment requires drastic deceleration during turns, which does not occur during curved paths. In contrast, stronger relationships between curved sprinting and COD speed could be observed using "shallower cuts" (i.e., angles of $30-45^{\circ}$ ) where there is a greater reliance on velocity maintenance and a reduction in braking requirements (Young et al., 2001). Not including other turning degrees (e.g., $45^{\mathrm{o}}$ degrees) could also be considered a study limitation. Additionally, it should be noted that the findings of this study are representative of semiprofessional soccer players; thus, caution is advised when generalizing these findings to athletes of different age-categories or performance levels. Future research is required to apply the COD and CS asymmetries to cutting tasks in a variety of cutting sports to provide information on how COD or CS asymmetries may differ between different levels and populations of athletes.

\section{Conclusion}

The main findings of this study reveal that CS and COD-90 abilities are moderately correlated, thus, should be evaluated and trained separately, at least for this population, when using these protocols. In addition, large relationships were reported between linear and multidirectional tasks (i.e., COD-90 $0^{\circ}$ and curve sprinting). These results indicate that performance in linear sprints is strongly related and greatly influences performance in multidirectional trajectories, whereas CS and COD- $90^{\circ}$ seem to be more independent actions. Therefore, practitioners should use COD, CS, and linear sprint tests as different testing categories, with the aim of obtaining a more complete picture of multidirectional speed performance. In terms of asymmetries, a new variable (CS asymmetry) could be considered in team-sport testing in order to detect players' magnitude and directions of inter-limb asymmetry and to establish the dominant curve side of each soccer player, which could contribute to better characterizing and profiling playing positions.

\section{Acknowledgements}

Thanks to all the participants in this study and to the soccer club.

\section{References}

Arcos AL, Aramendi JF, Emparanza JI, Castagna C, Yanci J, Lezáun A, Martínez-Santos R. Assessing Change of Direction Ability in a Spanish Elite Soccer Academy. J Hum Kinet, 2020; 72 229-239

Bishop C. Inter-limb Asymmetries : Are Thresholds a Usable Concept?. Strength Cond J, 2020; 1-13

Bishop C, Gonzalo-Skok O, Read P. Inter-Limb Asymmetry During Rehabilitation. Aspetar Sport Med J, 2020; (March): 18-22

Bishop C, Read P, Lake J, Chavda S, Turner A. Inter-Limb Asymmetries: Understanding How to Calculate Differences from Bilateral and Unilateral Tests. Strength Cond J, 2018; 1

Bloomfield J, Polman R, O'Donoghue P. Physical demands of different positions in FA Premier League soccer. J Sport Sci Med, 2007; 6(1): 63-70

Brughelli M, Cronin J, Levin G, Chaouachi A. Understanding change of direction ability in sport: A review of resistance training studies. Sport Med, 2008; 38(12): 1045-1063

Buttifant D, Graham K, Cross K. Agility and Speed of Soccer Players are Two Different Performance Parameters. J Sports Sci, 1999; 17809

Caldbeck P. Contextual Sprinting in Premier League, 2020; John Moores University, Liverpool

Chang YH, Kram R. Limitations to maximum running speed on flat curves. J Exp Biol, 2007; 210(6): 971-982

Çınarlı FS, Kafkas ŞA, Kafkas ME. Relationship Between Linear Running and Change of Direction Performances of Male Soccer Players. Turkish J Sport Exerc, 2018; 
Cuthbert M, Thomas C, Dos'Santos T, Jones PA. Application of Change of Direction Deficit to Evaluate Cutting Ability. J Strength Cond Res, 2019; 33(8): 2138-2144

Dos'Santos T, McBurnie A, Donelon T, Thomas C, Comfort P, Jones PA. A qualitative screening tool to identify athletes with "high-risk" movement mechanics during cutting: The cutting movement assessment score (CMAS). Phys Ther Sport, 2019; 38 152-161

Dos'Santos T, Thomas C, Jones PA, Comfort P. Assessing Asymmetries in Change of Direction Speed Performance; Application of Change of Direction Deficit. J Strength Cond Res, 2018; (January): 1

Dos'Santos T, Thomas C, Jones PA, Comfort P. Assessing Asymmetries in Change of Direction Speed Performance. J Strength Cond Res, 2019; 33(11): 2953-2961

Field A. Discovering Statistics using IBM SPSS Statistics - Cluster Analysis. Discov Stat Using IBM SPSS Stat, 2013; 297-321

Filter A, Olivares-Jabalera J, Santalla A, Morente-Sánchez J, Robles-Rodríguez J, Requena B, Loturco I. Curve Sprinting in Soccer: Kinematic and Neuromuscular Analysis. Int J Sports Med, 2020; 41(11): 744-750

Fílter A, Olivares J, Santalla A, Nakamura FY, Loturco I, Requena B. New curve sprint test for soccer players: Reliability and relationship with linear sprint. J Sports Sci, 2019; 1-6

Fitzpatrick JF, Linsley A, Musham C. Curved sprinting during football match-play Running the curve: a preliminary investigation into curved sprinting during football match-play. Sport Perform Sci Reports, 2019; 1(55): 1-3

Freitas TT, Jeffreys I, Reis VP, Fernandes V, Alcaraz PE, Pereira LA, Loturco I. Multidirectional sprints in soccer: are there connections between linear, curved, and change-of-direction speed performances?. J Sports Med Phys Fitness, 2020;

Hopkins WG, Marshall SW, Batterham AM, Hanin J. Progressive statistics for studies in sports medicine and exercise science. Med Sci Sports Exerc, 2009;

Jeffreys I, Huggins S, Davies N. Delivering a gamespeed-focused speed and agility development program in an english premier league soccer academy. Strength Cond J, 2018; 40(3): 23-32

Lakens D. Calculating and reporting effect sizes to facilitate cumulative science: A practical primer for t-tests and ANOVAs. Front Psychol, 2013; 4(NOV): 863

Little T, Williams AG. Specificity of Acceleration, Maximum Speed, and Agility in Professional Soccer Players. J Strength Cond Res, 2005; 19(1): 76

Loturco I, Pereira LA, Fílter A, Olivares-Jabalera J, Reis VP, Fernandes V, Freitas T, Requena B. Curve sprinting in soccer: Relationship with linear sprints and vertical jump performance. Biol Sport, 2020; 37(3): 277-283

Madruga-Parera M, Bishop C, Beato M, Fort-Vanmeerhaeghe A, Gonzalo-Skok O, Romero-Rodríguez D. Relationship Between Interlimb Asymmetries and Speed and Change of Direction Speed in Youth Handball Players. J Strength Cond Res, 2019; Publish Ah(1): 1

Madruga-Parera M, Bishop C, Read P, Lake J, Brazier J, Romero-Rodriguez D. Jumping-based Asymmetries are Negatively Associated with Jump, Change of Direction, and Repeated Sprint Performance, but not Linear Speed, in Adolescent Handball Athletes. J Hum Kinet, 2020; 71 47-58

Maloney SJ, Richards J, Nixon DGD, Harvey LJ, Fletcher IM. Do stiffness and asymmetries predict change of direction performance?. J Sports Sci, 2016; 0414(April): 1-10

Maszczyk A, Zajac A, Rygula I. A neural network model approach to athlete selection. Sports Engineering, 2011; 13(2): 83-93

Nimphius S. Increasing Agility.In D. Joyce \& D. Lewindon (Eds.), High-Performance Training for Sports, 2014; (Human Kine., pp. 185-197) Champaign, IL

Ohnuma H, Tachi M, Kumano A, Hirano Y. How to Maintain Maximal Straight Path Running Speed on a 
Curved Path in Sprint Events. J Hum Kinet, 2018; 62(1): 23-31

Raya-González J, Bishop C, Gómez-Piqueras P, Veiga S, Viejo-Romero D, Navandar A. Strength, Jumping, and Change of Direction Speed Asymmetries Are Not Associated With Athletic Performance in Elite Academy Soccer Players. Front Psychol, 2020; 11(March): 1-8

Rouissi M, Chtara M, Owen A, Chaalali A, Chaouachi A, Gabbett T, Chamari K. Effect of leg dominance on change of direction ability amongst young elite soccer players. J Sports Sci, 2016; 34(6): 542-548

Šimonek J, Horička P, Hianik J. The differences in acceleration, maximal speed and agility between soccer, basketball, volleyball and handball players. J Hum Sport Exerc, 2017; 12(1): 73-82

Suarez-Arrones L, Gonzalo-Skok O, Carrasquilla I, Asián-Clemente J, Santalla A, Lara-Lopez P, Núñez FJ. Relationships between Change of Direction, Sprint, Jump, and Squat Power Performance. Sports, 2020; 8(3): 38

Thomas J, Nelson JK. Research Methods in Physical ActivityChampaign, IL: Human Kinetics, 1990;

Turner A, Walker S, Stembridge M, Coneyworth P, Reed G, Birdsey L, Barter P, Moody J. A testing battery for the assessment of fitness in soccer players. Strength Cond J, 2011; 33(5): 29-39

Young W, McDowell M, Scarlett B. Specificity of sprint and agility training methods. J Strength Cond Res, 2001; 15(03): 315-319

Young WB, James R, Montgomery I. Is muscle power related to running speed with changes of direction?. J Sports Med Phys Fitness, 2002; 42(3): 282-288

\section{Corresponding author:}

\section{Alberto Filter, PhD}

Cañada Rosal 41439 (Seville), Spain.

Phone: +34954839024

Email: albertofr_91@hotmail.com 